\title{
Severity of obstructive sleep apnoea predicts coronary artery plaque burden: a coronary computed tomographic angiography study
}

\author{
Brian D. Kent ${ }^{1,2}$, John F. Garvey ${ }^{1,2}$, Silke Ryan ${ }^{1,2}$, Geraldine Nolan¹, \\ Jonathan D. Dodd ${ }^{3,4}$ and Walter T. McNicholas ${ }^{1,2,4}$
}

\begin{abstract}
Affiliations: ${ }^{1}$ Pulmonary and Sleep Disorders Unit, St Vincent's University Hospital, Dublin, ${ }^{2}$ School of Medicine and Medical Science, University College Dublin, Dublin, and ${ }^{3}$ Dept of Radiology, St Vincent's University Hospital, Dublin, Ireland. "Both authors contributed equally.
\end{abstract}

Correspondence: W.T. McNicholas, Pulmonary and Sleep Disorders Unit, St Vincent's University Hospital, Dublin 4, Ireland. E-mail: walter.mcnicholasducd.ie

ABSTRACT Obstructive sleep apnoea (OSA) is associated with significantly increased risk of cardiovascular disease. Carotid ultrasonography and retrospective, uncontrolled, coronary imaging studies have suggested an association of OSA with subclinical atherosclerosis, but there is a lack of prospective, controlled studies directly evaluating the relationship of OSA with occult coronary artery disease.

We performed coronary computed tomographic angiography and inpatient-attended sleep studies on a cohort of otherwise healthy males attending our sleep laboratory, and compared coronary artery plaque volume between subjects with low and high apnoea/hypopnoea index (AHI) scores.

29 subjects participated. The median AHI was 15.5 events $\cdot \mathrm{h}^{-1}$, with subjects who scored above this classified as high AHI. No significant differences were observed in demographic, anthropometric and clinical variables between the high- and low-AHI groups. Coronary plaque volume was significantly greater in the high-AHI group (mean plaque volume $2.6 \pm 0.7 \mathrm{~mm}^{2}$ versus $0.8 \pm 0.2 \mathrm{~mm}^{2} ; \mathrm{p}=0.017$ ) and, furthermore, correlated significantly with AHI (Spearman's $r=0.433 ; p=0.019$ ). Following adjustment for dyslipidaemia and fasting plasma glucose levels, AHI remained a significant predictor of plaque volume (standardised $\beta=0.424 ; p=0.027$ ).

In this prospective case-control study, we found that severity of OSA may predict occult coronary atherosclerosis in otherwise healthy overweight or obese male subjects.

@ERSpublications

Increasing OSA severity predicts a greater burden of occult coronary artery disease in healthy overweight or obese males http://ow.ly/nSXEU

Received: June 142012 | Accepted after revision: Dec 152012 | First published online: March 072013

Support statement: B.D. Kent and S. Ryan are funded by a grant from the Health Research Board (Dublin, Ireland) (HPF/ 2009/033).

Conflict of interest: None declared.

Copyright @ERS 2013 


\section{Introduction}

Obstructive sleep apnoea (OSA) is a highly prevalent disorder associated with diminished quality of life, reduced daytime cognitive functioning and increased risk of road traffic accidents. More important from a public health perspective, however, is its impact on cardiovascular morbidity and mortality [1]. Subjects with severe OSA are more likely to experience cardiovascular morbidity or die of cardiovascular causes than those without OSA $[2,3]$, and successful use of nocturnal continuous positive airway pressure (nCPAP) therapy is associated with a reduction in this increased risk of cardiovascular disease [4].

OSA and associated intermittent hypoxia are potent drivers of an atherogenic milieu, with evidence from population, clinical, animal and in vitro studies demonstrating their role in the development of systemic inflammation [5], oxidative stress [6], endothelial dysfunction [7] and metabolic dysfunction [8]. Furthermore, studies utilising carotid ultrasonography have shown severe OSA to be associated with increased carotid intima medial thickness (IMT), a validated early marker of coronary atherosclerosis [9]. Meanwhile, direct evidence supporting a deleterious impact of OSA on the coronary circulation comes from observational studies of clinic patients assessing coronary artery plaque burden using intravascular ultrasound [10], and coronary artery calcification using coronary computed tomographic angiography (CTA) [11].

However, the ability to discern a robust independent effect of OSA on cardiovascular outcomes in clinic population studies is limited by the high prevalence of comorbid confounding factors such as obesity and metabolic dysfunction. Moreover, it has been suggested that increased carotid IMT in OSA patients may be enhanced by snoring [12], while the data from studies directly examining the coronary circulation are compromised by a number of factors, but particularly a selection bias toward subjects with pre-existing coronary artery disease $[10,11]$.

Consequently there is a need for prospective data in well-controlled cohorts, free of comorbidities or other potential confounding factors, to assess the independent impact of sleep disordered breathing on coronary artery disease. This is facilitated by the validation of current coronary CTA as a measure of coronary atherosclerosis [13], thus permitting the accurate, noninvasive measurement of coronary artery plaque volume and composition. We assessed the impact of a diagnosis of OSA on subclinical coronary atherosclerosis in an otherwise healthy cohort of overweight or obese male subjects.

\section{Methods}

Subjects

Male subjects referred to our sleep clinic with symptoms of sleep disordered breathing, including snoring, witnessed apnoea during sleep and daytime somnolence, who were nonsmokers and free of any other medical disorder, were considered for enrolment in the study. To expedite subject enrolment, in parallel to patient recruitment, a small group of four normal male subjects were also recruited from hospital staff using advertisements placed throughout the hospital. Subjects were considered for participation in the study if they were aged 35-60 years, with a body mass index (BMI) $25-45 \mathrm{~kg} \cdot \mathrm{m}^{-2}$. The principal exclusion criteria were a history of coronary artery or cardiac disease, diabetes mellitus, chronic obstructive pulmonary disease, known dyslipidaemia or hypertension requiring treatment, smoking in the preceding 5 years or a $>10$ pack-years history of smoking. Subjects with known sleep disordered breathing on nCPAP were also excluded from the study. Research ethics committee approval was obtained before commencement of the study, and all subjects provided informed consent.

\section{Procedures}

Following enrolment, all study subjects were admitted overnight to the sleep laboratory for inpatient, attended cardiorespiratory polygraphy. Following the American Academy of Sleep Medicine 2007 rules [14], an apnoea event was scored if there was a $\geqslant 80 \%$ decrease in the amplitude of airflow during sleep, with a hypopnoea event scored by the combination of $a \geqslant 30 \%$ reduction in airflow with $a \geqslant 4 \%$ reduction of oxygen saturation. A minimum event duration of $10 \mathrm{~s}$ was required for apnoea or hypopnoea [14]. Following expert-reader interpretation of sleep studies, the study population was dichotomised, according to apnoea/hypopnoea index (AHI), into low or high AHI groups. Subjects with an AHI above the median were categorised as high AHI, with the remainder categorised as low AHI.

Self-reported sleepiness was assessed using the Epworth sleepiness scale. Blood pressure was measured in the sleep laboratory following $10 \mathrm{~min}$ in a recumbent position. Fasting blood samples including fasting plasma glucose, fasting lipids and glycosylated haemoglobin (HbA1c) were drawn the morning after the sleep study. Framingham risk scores were calculated based on the subjects' age, lipid profile and systolic blood pressure measurements. 


\section{Multi-detector computed tomography protocol}

Coronary CTA scans were obtained using a 64-slice multi-detector computed tomography (CT) scanner (Sensation 64; Siemens Medical Solutions, Erlangen, Germany) using image reconstruction with a slice thickness of $0.6 \mathrm{~mm}$ and $0.3 \mathrm{~mm}$ increments, and a dedicated cardiac field of view from the carina to the cardiac apex. A $\beta$-blocker was administered to patients with a heart rate $>60 \mathrm{bpm} 1 \mathrm{~h}$ before the coronary CTA examination. Glyceryl trinitrate was administered sublingually just prior to scan acquisition. Following a timing bolus, images were acquired during a single breath hold mid inspiration and administration of iodinated contrast. Images were acquired in a craniocaudal direction. Retrospective ECG-gated reconstructions at $60-70 \%$ of the $\mathrm{R}-\mathrm{R}$ interval were utilised to obtain coronary image sets with no or minimal motion artefact [15], with additional reconstructions if excessive motion artefact was present. All images were analysed on a three-dimensional workstation (Leonardo; Siemens Medical Solutions).

\section{Image analysis of coronary plaque}

Coronary CTA was analysed by a single expert reader (J.D. Dodd), who was blinded to the subjects' clinical characteristics and sleep study results. The following morphological features and techniques for plaque analysis were derived from previous studies [16-19]: plaque localisation within the coronary tree, plaque volume, plaque type and mean and minimal CT density. Plaque localisation within the coronary tree was described by documenting the major coronary artery (left main, left anterior descending, left circumflex and right coronary artery) and its specific coronary segment using standardised coronary CTA reporting nomenclature [15].

Plaque volume measurements were performed using a previously described technique [20]. Plaque type was classified into four categories according to previously published studies [20, 21], ranging from calcified (type 1) to purely noncalcified (type 4). Mean and minimal CT attenuations of the respective lesions were assessed in Hounsfield units (HU). Minimal CT attenuation within the plaque was measured using several circular regions of interest placed in the centre of the plaque at the site of maximal lumen narrowing. The lowest mean CT density of the regions of interest was defined as minimal CT density. At the same level, the total plaque area was manually traced and the displayed CT density within the manually assessed area was defined as mean CT density of the respective plaque.

\section{Outcome measures and statistical analysis}

The primary outcome measures were comparison of overall coronary artery plaque burden between subjects with low and high AHI scores, and the correlation of coronary plaque volume with indices of OSA severity. Secondary outcome measures included comparison of plaque distribution, number of vessels involved, and plaque density between the two groups.

Baseline characteristics of the two groups were compared using independent t-tests, the Mann-Whitney U-test or Chi-squared test as appropriate. Independent samples t-test was used to compare plaque burden and plaque density between the low and high AHI groups. Relationships between plaque burden and AHI, subjective sleepiness and demographic and anthropometric factors were assessed using Pearson's or Spearman's correlation coefficient.

Factors with a significant relationship with plaque volume in univariate regression analysis were incorporated into an exploratory stepwise multivariate regression model using plaque volume as the dependent variable. Non-normally distributed values were log-transformed for incorporation into this model. Coronary artery plaque distribution and the number of coronary vessels involved were assessed by Chi-squared tests.

Based on previous studies, a sample size of 12 subjects per group was estimated to provide $80 \%$ power to detect a $60-\mathrm{mm}^{3}$ difference in mean plaque volume between groups, with a two-sided $\alpha=0.05$ [10].

\section{Results}

\section{Patient characteristics}

29 overweight or obese male subjects were studied; 25 recruited from our sleep clinic and four healthy hospital staff members. Subject characteristics are summarised in table 1 . The mean $\pm \mathrm{SD}$ age was $44.9 \pm 7.5$ years and mean \pm SD BMI $32.7 \pm 4.8 \mathrm{~kg} \cdot \mathrm{m}^{-2}$, with $69 \%$ of the cohort being obese (BMI $\geqslant 30 \mathrm{~kg} \cdot \mathrm{m}^{-2}$ ). $31 \%$ of subjects had no OSA (AHI $<5$ events $\cdot \mathrm{h}^{-1}$ ), $27.6 \%$ mild-to-moderate OSA (AHI 5-30 events $\cdot \mathrm{h}^{-1}$ ) and $41.4 \%$ severe OSA (AHI $\geqslant 30$ events $\cdot \mathrm{h}^{-1}$ ). The cohort was considered to be at low risk of cardiovascular disease by conventional measures, with a mean \pm SD 10 -year Framingham risk score of $3.4 \pm 3.2 \%$. 31\% were former smokers. The median AHI was 15.5 events $\cdot \mathrm{h}^{-1}$, with subjects scored at or below this classified as low AHI, and above this as high AHI. $60 \%$ of the low-AHI group had no OSA, while $85.7 \%$ of the high-AHI group were classified as having severe OSA. Compared with the low-AHI 


\section{TABLE 1 Patient characteristics}

\begin{tabular}{|c|c|c|c|c|}
\hline & Overall & Low AHI & High AHI & p-value \\
\hline Subjects n & 29 & 15 & 14 & \\
\hline Age years & $44.9 \pm 7.5$ & $45.1 \pm 8.6$ & $44.6 \pm 6.4$ & 0.842 \\
\hline $\mathrm{BMI} \mathrm{kg} \cdot \mathrm{m}^{-2}$ & $32.7 \pm 4.8$ & $32.2 \pm 4.1$ & $33.3 \pm 5.6$ & 0.529 \\
\hline Obese & $6 \overline{9}$ & 66.7 & 71.4 & 0.781 \\
\hline ESS score & $10.6 \pm 5.6$ & $7.7 \pm 4.8$ & $13.2 \pm 5.1$ & 0.007 \\
\hline AHI events $\cdot h^{-1}$ & $25.0 \pm 25.0$ & $5.5 \pm 5.5$ & $45.8 \pm 20.1$ & $<0.001$ \\
\hline ODI events $\cdot h^{-1}$ & $22.3 \pm 23.4$ & $4.2 \pm 4.1$ & $41.6 \pm 22.0$ & $<0.001$ \\
\hline Ex-smoker & 31 & 26.7 & 35.7 & 0.91 \\
\hline Systolic BP mmHg & $130.7 \pm 13.2$ & $130.7 \pm 13.9$ & $130.6 \pm 13.2$ & 0.996 \\
\hline Diastolic BP mmHg & $83.4 \pm 9.6$ & $83.2 \pm 10.2$ & $83.6 \pm 9.3$ & 0.919 \\
\hline Fasting plasma glucose $\mathrm{mmol} \cdot \mathrm{L}^{-1}$ & $5.52 \pm 1.09$ & $5.58 \pm 1.11$ & $5.48 \pm 1.11$ & 0.833 \\
\hline HbA1c \% & $5.82 \pm 0.57$ & $5.76 \pm 0.53$ & $5.87 \pm 0.62$ & 0.626 \\
\hline Total cholesterol $\mathrm{mmol} \cdot \mathrm{L}^{-1}$ & $4.98 \pm 0.77$ & $5.27 \pm 0.77$ & $4.71 \pm 0.69$ & 0.061 \\
\hline $\mathrm{HDL}$-cholesterol $\mathrm{mmol} \cdot \mathrm{L}^{-1}$ & $1.04 \pm 0.22$ & $1.12 \pm 0.22$ & $0.97 \pm 0.21$ & 0.09 \\
\hline Framingham risk score & $3.4 \pm 3.2$ & $3.4 \pm 3.1$ & $3.4 \pm 3.4$ & 0.972 \\
\hline
\end{tabular}

Data are presented as mean \pm SD or \%, unless otherwise stated. AHI: apnoea/hypopnoea index; BMI: body mass index; ESS: Epworth sleepiness scale; ODI: oxyhaemoglobin desaturation index; BP: blood pressure; HbA1c: glycosylated haemoglobin; HDL: high-density lipoprotein.

group, subjects in the high-AHI group were sleepier, but no significant differences in age, BMI, smoking history, systolic or diastolic blood pressure, fasting plasma glucose, HbAlc levels, fasting lipids or 10-year Framingham risk scores were observed between the two groups (table 1).

\section{Coronary artery plaque volume}

Subjects in the high-AHI group had a significantly higher coronary artery plaque burden than those in the low-AHI group (mean \pm SD plaque volume $2.6 \pm 0.7 \mathrm{~mm}^{3}$ versus $0.8 \pm 0.2 \mathrm{~mm}^{3} ; \mathrm{p}=0.017$ ) (fig. 1). Plaque volume correlated significantly with AHI (Spearman's $\mathrm{r}=0.433 ; \mathrm{p}=0.019$ ), oxyhaemoglobin desaturation index (Spearman's $r=0.387 ; \mathrm{p}=0.038$ ), 10-year Framingham risk score (Spearman's $r=0.402 ; \mathrm{p}=0.037$ ), fasting plasma glucose (Spearman's $\mathrm{r}=0.407 ; \mathrm{p}=0.035$ ) and high-density lipoprotein (HDL)-cholesterol (Spearman's $\mathrm{r}=-0.481 ; \mathrm{p}=0.013)$. No significant correlations with plaque volume were seen for BMI, age, daytime sleepiness, systolic or diastolic blood pressure measurements, or HbA1c levels (table 2). Stepwise multivariate regression analysis revealed an independent relationship between coronary artery plaque volume and AHI following adjustment for fasting plasma glucose and HDL-cholesterol (model $\mathrm{R}^{2}=0.21$, standardised $\beta=0.424 ; p=0.027$ ) (table 3 ). Representative coronary CTA images from study subjects are demonstrated in figure 2.

\section{Coronary artery plaque distribution}

$44.8 \%$ of subjects had plaque identified in the proximal segments of the left anterior descending or right coronary arteries, $26.7 \%$ of the low-AHI group and $64.3 \%$ of the high-AHI group (Chi-squared $\mathrm{p}=0.042$ ).

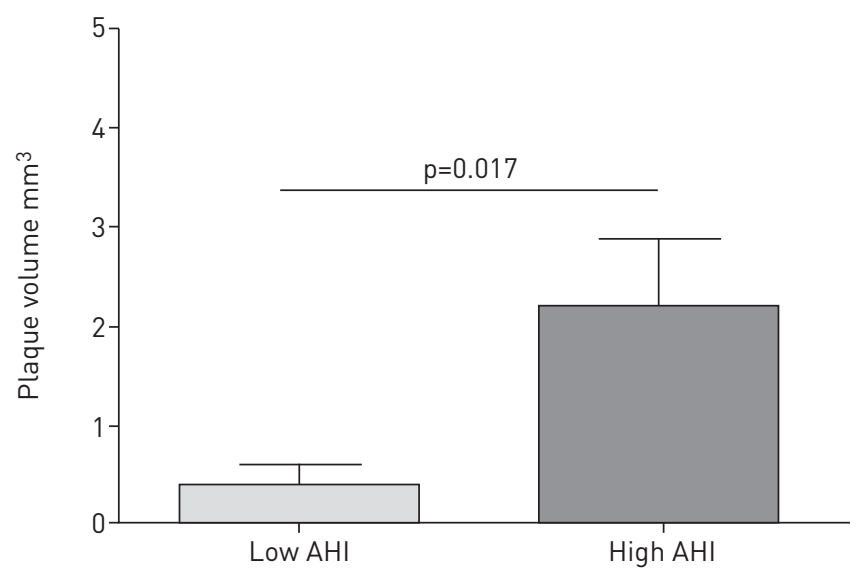

FIGURE 1 Mean plaque volume according to severity of obstructive sleep apnoea. AHI: apnoea/hypopnoea index. 
TABLE 2 Correlations with plaque volume

Correlation coefficient

\begin{tabular}{lcc}
\hline AHI & 0.433 & 0.019 \\
ODI & 0.387 & 0.038 \\
BMI & 0.133 & 0.493 \\
Age & 0.321 & 0.089 \\
ESS score & 0.119 & 0.553 \\
Fasting plasma glucose & 0.407 & 0.035 \\
Total cholesterol & 0.017 & 0.934 \\
HDL-cholesterol & -0.434 & 0.024 \\
Systolic BP & 0.216 & 0.261 \\
Diastolic BP & 0.304 & 0.109 \\
Framingham risk score & 0.402 & 0.037 \\
\hline
\end{tabular}

AHI: apnoea/hypopnoea index; ODI: oxyhaemoglobin desaturation index; BMI: body mass index; ESS: Epworth sleepiness scale; HDL: high-density lipoprotein; BP: blood pressure.

In subjects with coronary artery plaque, the mean \pm SD number of vessels affected was $1.3 \pm 0.9$, with no difference identified between the low- and high-AHI groups $(1.5 \pm 1.2$ versus $1.2 \pm 0.6 ; \mathrm{p}=0.525)$.

\section{Coronary artery plaque density}

In the overall cohort, $48.3 \%$ of the subjects with coronary artery plaque had low-density plaques. There was a slight trend towards a greater number of soft plaques in the high-AHI compared to the low-AHI group ( $50 \%$ versus $33.3 \%$; $\mathrm{p}=0.696$ ).

No significant difference was observed between the two groups in mean plaque density $(331.6 \pm 229.3 \mathrm{HU}$ versus $245.4 \pm 219.7 \mathrm{HU} ; \mathrm{p}=0.467)$ or lowest plaque density $(369 \pm 247 \mathrm{HU}$ versus $287 \pm 255 \mathrm{HU}$; $\mathrm{p}=0.536)$.

\section{Discussion}

In this first prospective study directly examining the independent effects of sleep disordered breathing on the coronary circulation in otherwise healthy males, we found a significant relationship between OSA severity and the presence and volume of subclinical coronary atherosclerosis. Moreover, subjects with severe OSA were more likely to have measurable lesions in more proximal coronary segments. There was no significant difference between the high- and low-AHI groups with regard to BMI, the proportion of obese individuals, or 10-year Framingham risk scores, so these findings appear to be independent of conventional cardiovascular risk markers.

A growing body of evidence suggests an independent role for sleep disordered breathing in driving atherosclerosis and subsequent clinically overt cardiovascular disease. A diagnosis of OSA is independently associated with both prevalent and incident hypertension [22], as well as incident coronary artery disease [23] and stroke risk [24]. Moreover, patients with severe OSA are significantly more likely to die of cardiovascular causes than those without OSA [2], and while the absolute risk increase attributable to sleep disordered breathing may be relatively small at an individual level, its high prevalence makes it an important contributor to cardiovascular morbidity and mortality at a population level [25]. Previous reports have suggested that sleep disordered breathing may substantially contribute to coronary atherogenesis [26] and worse outcomes in subjects with established coronary artery disease [27].

\begin{tabular}{lccc} 
TABLE 3 Multivariate regression model of predictors of coronary artery plaque volume \\
\cline { 2 - 2 } & \multicolumn{1}{c}{ Standardised $\boldsymbol{\beta}$} & $\mathbf{\beta}$ (95\% Cl) & $\mathbf{p}$-value \\
\hline AHI & 0.424 & $0.036(0.004-0.07)$ & 0.027 \\
Fasting plasma glucose & 0.120 & $0.128(-0.68-0.93)$ & 0.745 \\
HDL-cholesterol & -0.144 & $-1.40(-5.67-2.87)$ & 0.505
\end{tabular}

Dependent variable: plaque volume. Model $\mathrm{R}^{2}$ : 0.21. AHI: apnoea/hypopnoea index; HDL: high-density lipoprotein. 

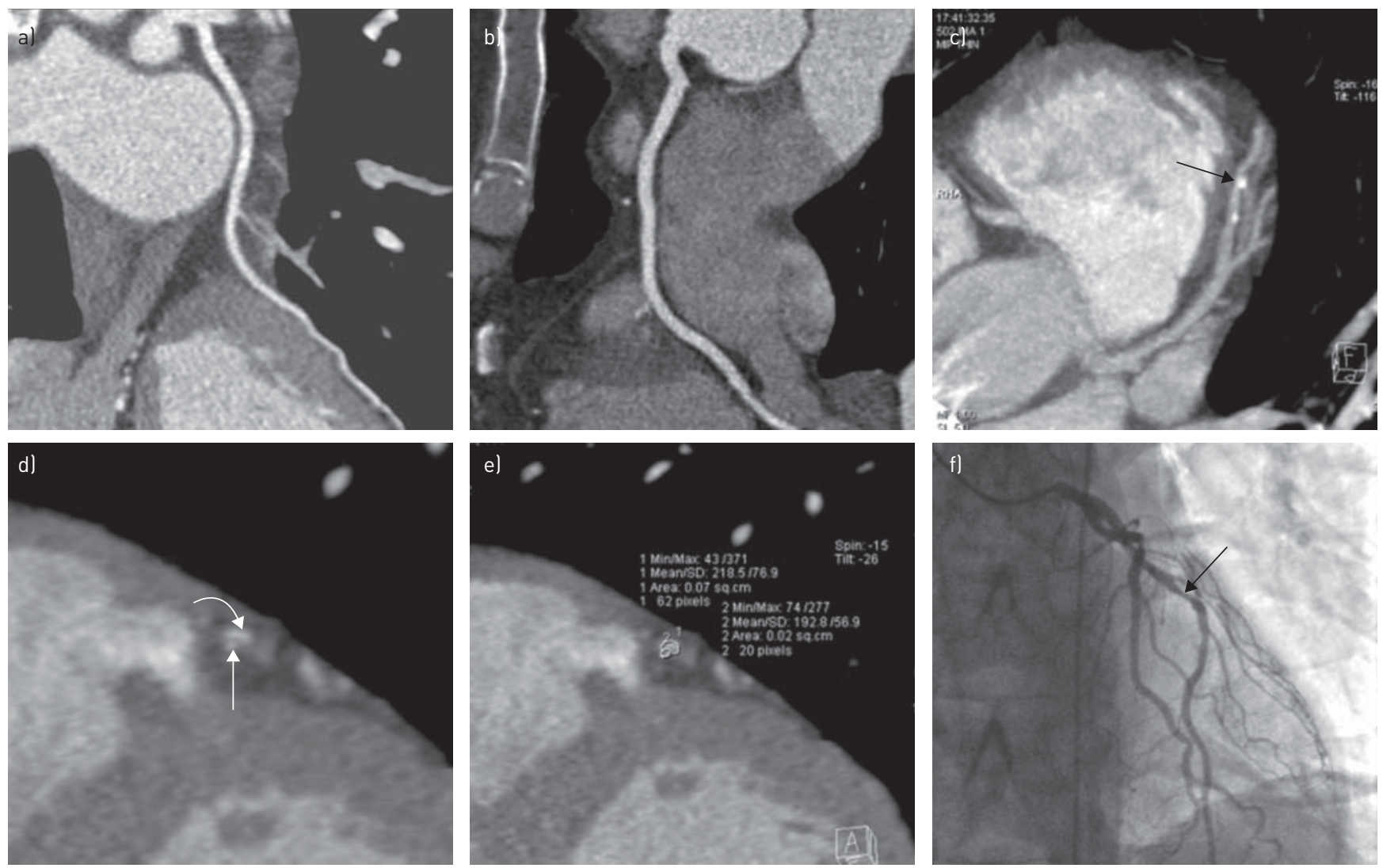

FIGURE 2 Coronary computed tomographic angiography images from study subjects. a) left anterior descending (LAD) healthy control; b) right coronary artery healthy control; c) LAD of obstructive sleep apnoea patient with distal calcified plaque (arrow); d) 1-mm cross-sectional slice through the distal LAD plaque, showing a patent lumen (straight arrow) and the plaque (curved arrow); e) calculation of the plaque volume; f) invasive coronary angiogram of the same patient confirming an obstructive distal LAD stenosis (arrow).

That OSA should be associated with increased coronary artery plaque burden should not be surprising, given its ability to generate a pro-atherogenic molecular milieu. Robust evidence supports a key role for OSA and associated intermittent hypoxia in driving systemic inflammation [5], oxidative stress [6] and endothelial dysfunction [7], all upstream precursors to atherosclerosis. Furthermore, there is increasing evidence to suggest that OSA and intermittent hypoxia are independently associated with metabolic dysfunction [8]. Meanwhile, compelling recent data demonstrates the ability of a model of intermittent hypoxia to cause hypertension even in healthy subjects [28].

To date, however, there has been a relative lack of prospective data directly examining the effects of OSA on the coronary circulation and previous reports on this topic have been compromised by a number of factors. For example, a large retrospective study of subjects undergoing coronary CTA and polysomnography (PSG) in close temporal proximity found that OSA severity was a strong independent predictor of coronary artery calcification [11]. However, subjects assessed in this study had underlying clinical indications for examination of the coronary vasculature, and had a substantial burden of cardiometabolic comorbidity. Similarly, an intravascular ultrasound study of subjects with stable coronary artery disease also suggested a significant relationship between AHI and coronary atherosclerotic plaque volume [10]. Again, these data were limited by the focus on subjects with known coronary artery disease.

Perhaps the best prospective evidence in this field, to date, comes from indirect assessment of coronary atherosclerosis obtained by measurement of carotid IMT. Carotid IMT is a validated surrogate of coronary artery disease in general populations, and has been assessed prospectively in otherwise healthy OSA subjects. Both Minoguchi et al. [29] and Drager et al. [9] found increased carotid IMT was predicted by increasing severity of sleep disordered breathing, with the latter group further demonstrating that successful initiation of nCPAP therapy lead to partial abrogation of this increase [30]. Conversely, evidence from population studies such as the Sleep Heart Health study and data from animal models suggest that any increase in carotid IMT in OSA patients may be attributable to traditional cardiovascular risk factors, or to 
snoring-related acoustic vibrations, rather than to sleep disordered breathing per se [12, 31]. Consequently, confirmatory data from direct examination of the coronary circulation remains highly desirable.

We utilised coronary CTA as the primary imaging method for assessing plaque volume. Several studies have shown a robust agreement between plaque detection and quantification with coronary CTA and invasive gold-standards such as intravascular ultrasound [16, 32]. That almost half our cohort demonstrated noncalcified plaques highlights an important advantage of coronary CTA over calcium scoring alone, suggesting that asymptomatic OSA patients undergoing cardiovascular evaluation should have coronary CTA rather than calcium scoring if true plaque burden is to be assessed. Other studies have also found substantial plaque burden and a negative coronary calcium score in at-risk groups [33]. Although conjectural, our finding of a substantially higher prevalence of plaques in proximal coronary segments in high-AHI patients may have outcome implications, such proximal plaques are strongly associated in other high-risk groups with subsequent adverse cardiac events [34].

The principal limitation of our study is the small cohort size; however, this is significantly offset by the prospective nature of the study, and the exclusion of subjects with known cardiometabolic comorbidities and smokers. Furthermore, although not powered to detect differences in our secondary outcome parameters, the study was adequately powered to demonstrate a significant difference in subclinical coronary artery plaque burden. Another potential limitation was the use of cardiorespiratory polygraphy over PSG, thereby underestimating true AHI. However, previous authors have demonstrated that subjects with an AHI in the normal range in a polygraphic study are unlikely to be diagnosed with clinically significant OSA on PSG [35]. Our patients were in many ways characteristic of the typical sleep clinic patient, male, middle-aged and overweight, but the absence of the usual comorbidities seen in a clinic population enhances the relevance of these findings outside of this context. Nonetheless, further studies are required to assess if our findings hold true for larger, less selected populations.

In summary, in a pilot study of otherwise healthy male sleep clinic patients and obese male controls, we found that coronary artery plaque volume as measured by coronary CTA correlated significantly with the degree of sleep disordered breathing, with higher plaque burden seen in subjects with more severe OSA. Future studies should explore the generalisability of these findings to larger, sleep clinic and communitybased populations, and to examine the reversibility of coronary atherosclerosis with nCPAP therapy.

\section{Acknowledgements}

The authors wish to acknowledge the input of Cormac Taylor (School of Medicine and Medical Science, University College Dublin, Dublin, Ireland) along with the contribution of Lynda Hayes, Audrey Russell and the staff in the Pulmonary and Sleep Disorders Unit, St Vincent's University Hospital (Dublin, Ireland) to this study.

\section{References}

1 McNicholas WT, Bonsigore MR. Sleep apnoea as an independent risk factor for cardiovascular disease: current evidence, basic mechanisms and research priorities. Eur Respir J 2007; 29: 156-178.

2 Punjabi NM, Caffo BS, Goodwin JL, et al. Sleep-disordered breathing and mortality: a prospective cohort study. PLoS Med 2009; 6: e1000132.

3 Gottlieb DJ, Yenokyan G, Newman AB, et al. Prospective study of obstructive sleep apnea and incident coronary heart disease and heart failure: the Sleep Heart Health Study. Circulation 2010; 122: 352-360.

4 Marin JM, Carrizo SJ, Vicente E, et al. Long-term cardiovascular outcomes in men with obstructive sleep apnoeahypopnoea with or without treatment with continuous positive airway pressure: an observational study. Lancet 2005; 365: 1046-1053

5 Ryan S, Taylor CT, McNicholas WT. Selective activation of inflammatory pathways by intermittent hypoxia in obstructive sleep apnea syndrome. Circulation 2005; 112: 2660-2667.

6 Lavie L, Lavie P. Molecular mechanisms of cardiovascular disease in OSAHS: the oxidative stress link. Eur Respir J 2009; 33: 1467-1484.

7 Jelic S, Padeletti M, Kawut SM, et al. Inflammation, oxidative stress, and repair capacity of the vascular endothelium in obstructive sleep apnea. Circulation 2008; 117: 2270-2278.

8 Punjabi NM, Shahar E, Redline S, et al. Sleep-disordered breathing, glucose intolerance, and insulin resistance: the Sleep Heart Health Study. Am J Epidemiol 2004; 160: 521-530.

9 Drager LF, Bortolotto LA, Lorenzi MC, et al. Early signs of atherosclerosis in obstructive sleep apnea. Am J Respir Crit Care Med 2005; 172: 613-618.

10 Turmel J, Sériès F, Boulet LP, et al. Relationship between atherosclerosis and the sleep apnea syndrome: an intravascular ultrasound study. Int J Cardiol 2009; 132: 203-209.

11 Sorajja D, Gami AS, Somers VK, et al. Independent association between obstructive sleep apnea and subclinical coronary artery disease. Chest 2008; 133: 927-933.

12 Cho JG, Witting PK, Verma M, et al. Tissue vibration induces carotid artery endothelial dysfunction: a mechanism linking snoring and carotid atherosclerosis? Sleep 2011; 34: 751-757.

13 Taylor AJ, Cerqueira M, Hodgson JM, et al. ACCF/SCCT/ACR/AHA/ASE/ASNC/NASCI/SCAI/SCMR 2010 appropriate use criteria for cardiac computed tomography. A report of the American College of Cardiology Foundation Appropriate Use Criteria Task Force, the Society of Cardiovascular Computed Tomography, the American College of Radiology, the American Heart Association, the American Society of Echocardiography, the American Society of Nuclear Cardiology, the North American Society for Cardiovascular Imaging, the Society for 
Cardiovascular Angiography and Interventions, and the Society for Cardiovascular Magnetic Resonance. Circulation 2010; 122: e525-e555.

14 Iber C, Ancoli-Israel S, Chesson AL, et al. The AASM Manual for the Scoring of Sleep and Associated Events: Rules, Terminology and Technical Specifications. 1st Edn. Westchester, American Academy of Sleep Medicine, 2007.

15 Raff GL, Abidov A, Achenbach S, et al. SCCT guidelines for the interpretation and reporting of coronary computed tomographic angiography. J Cardiovasc Comput Tomogr 2009; 3: 122-136.

16 Pflederer T, Marwan M, Schepis T, et al. Characterization of culprit lesions in acute coronary syndromes using coronary dual-source CT angiography. Atherosclerosis 2010; 211: 437-444.

17 Schepis T, Marwan M, Pflederer T, et al. Quantification of non-calcified coronary atherosclerotic plaques with dual-source computed tomography: comparison with intravascular ultrasound. Heart 2010; 96: 610-615.

18 Lin F, Shaw LJ, Berman DS, et al. Multidetector computed tomography coronary artery plaque predictors of stressinduced myocardial ischemia by SPECT. Atherosclerosis 2008; 197: 700-709.

19 Min JK, Edwardes M, Lin FY, et al. Relationship of coronary artery plaque composition to coronary artery stenosis severity: results from the prospective multicenter ACCURACY trial. Atherosclerosis 2011; 219: 573-578.

20 Leber AW, Knez A, von Ziegler F, et al. Quantification of obstructive and nonobstructive coronary lesions by 64slice computed tomography: a comparative study with quantitative coronary angiography and intravascular ultrasound. J Am Coll Cardiol 2005; 46: 147-154.

21 Weigold WG, Abbara S, Achenbach S, et al. Standardized medical terminology for cardiac computed tomography: a report of the Society of Cardiovascular Computed Tomography. J Cardiovasc Comput Tomogr 2011; 5: 136-144.

22 Peppard PE, Young T, Palta M, et al. Prospective study of the association between sleep-disordered breathing and hypertension. N Engl J Med 2000; 342: 1378-1384.

23 Peker Y, Carlson J, Hedner J. Increased incidence of coronary artery disease in sleep apnoea: a long-term follow-up. Eur Respir J 2006; 28: 596-602.

24 Mooe T, Franklin KA, Holmström K, et al. Sleep-disordered breathing and coronary artery disease: long-term prognosis. Am J Respir Crit Care Med 2001; 164: 1910-1913.

25 Young T, Palta M, Dempsey J, et al. The occurrence of sleep-disordered breathing among middle-aged adults. N Engl J Med 1993; 328: 1230-1235.

26 Hayashi M, Fujimoto K, Urushibata K, et al. Nocturnal oxygen desaturation correlates with the severity of coronary atherosclerosis in coronary artery disease. Chest 2003; 124: 936-941.

27 Peker Y, Hedner J, Kraiczi H, et al. Respiratory disturbance index: an independent predictor of mortality in coronary artery disease. Am J Respir Crit Care Med 2000; 162: 81-86.

28 Tamisier R, Pépin JL, Rémy J, et al. 14 nights of intermittent hypoxia elevate daytime blood pressure and sympathetic activity in healthy humans. Eur Respir J 2011; 37: 119-128.

29 Minoguchi K, Yokoe T, Tazaki T, et al. Increased carotid intima-media thickness and serum inflammatory markers in obstructive sleep apnea. Am J Respir Crit Care Med 2005; 172: 625-630.

30 Drager LF, Bortolotto LA, Figueiredo AC, et al. Effects of continuous positive airway pressure on early signs of atherosclerosis in obstructive sleep apnea. Am J Respir Crit Care Med 2007; 176: 706-712.

31 Wattanakit K, Boland L, Punjabi NM, et al. Relation of sleep-disordered breathing to carotid plaque and intimamedia thickness. Atherosclerosis 2008; 197: 125-131.

32 Achenbach S, Ropers D, Kuettner A, et al. Contrast-enhanced coronary artery visualization by dual-source computed tomography - initial experience. Eur J Radiol 2006; 57: 331-335.

33 Villines TC, Hulten EA, Shaw LJ, et al. Prevalence and severity of coronary artery disease and adverse events among symptomatic patients with coronary artery calcification scores of zero undergoing coronary computed tomography angiography: results from the CONFIRM (Coronary CT Angiography Evaluation for Clinical Outcomes: An International Multicenter) registry. J Am Coll Cardiol 2011; 58: 2533-2540.

34 Budoff MJ, Dowe D, Jollis JG, et al. Diagnostic performance of 64-multidetector row coronary computed tomographic angiography for evaluation of coronary artery stenosis in individuals without known coronary artery disease: results from the prospective multicenter ACCURACY (Assessment by Coronary Computed Tomographic Angiography of Individuals Undergoing Invasive Coronary Angiography) trial. J Am Coll Cardiol 2008; 52: 1724-1732.

35 Dingli K, Coleman EL, Vennelle M, et al. Evaluation of a portable device for diagnosing the sleep apnoea/ hypopnoea syndrome. Eur Respir J 2003; 21: 253-259. 